\title{
The Ontogeny of Sugar Transport in Kidney
}

\author{
KARL S. ROTH, SHING MEI HWANG, MARC YUDKOFF, AND STANTON SEGAL ${ }^{(23)}$ \\ Division of Biochemical Development and Molecular Diseases, Children's Hospital of Philadelphia, and the \\ Departments of Pediatrics and Medicine, University of Pennsylvania School of Medicine, Philadelphia, Pennsylvania, \\ $U S A$
}

\section{Summary}

Concentrative uptake of $\alpha$-methyl-D-glucoside (AMG) by isolated renal tubule fragments from the newborn Sprague-Dawley rat has been demonstrated and the validity of this phenomenon confirmed by an in vivo demonstration of AMG uptake by the newborn kidney cortex. A kinetic analysis of the entry phenomenon in the newborn tubule reveals the presence of two distinct membrane transport systems for AMG, only one of which is present in the adult tubule. All transport of sugar in both newborn and adult tubules was phlorizin-sensitive, but was only partially inhibited in $\mathrm{Na}^{+}$-free buffer. Glucose was shown to inhibit uptake competitively on the shared, high capacity system. Uptake on the high affinity system in the newborn represents $15-20 \%$ of the total at physiologic sugar concentrations. It is concluded that active sugar transport is a characteristic of the newborn rat kidney and that the isolated tubule preparation is a more accurate reflection of this phenomenon than is the renal cortical slice.

\section{Speculation}

The presence of a unique high affinity glucose transport system in newborn renal tubules in addition to the lower affinity system also found in the adult enables the anatomically immature kidney to efficiently reclaim sugar from the glomerular filtrate and, thus, to prevent loss of calorically valuable nutrients in the promotion of growth.

Sugar transport by the renal tubule has been the subject of a vast number of investigations extending over many years (19). The results of in vitro studies sometimes have been difficult to correlate with the in vivo situation. An example of this is the transport of hexoses by newborn rat kidney. Investigations employing the renal cortical slice of newborn rat kidney failed to demonstrate either galactose accumulation (2) or AMG uptake $(16,17)$. Both sugars are known to share the glucose transport system(s) in adult rat kidney. Although this lack of uptake in vitro might be expected to result in neonatal glycosuria in vivo, Segal et al. (15) were unable to detect glucose in bladder urine from newborn Sprague-Dawley rats. The mechanism by which the neonatal kidney removes hexoses from the glomerular ultrafiltrate (which would resolve these paradoxical observations) had been left unexamined until the recent demonstration of active uptake of $\mathrm{AMG}$ by isolated renal proximal tubule fragments prepared from newborn Sprague-Dawley rat (13). This study demonstrates the in vivo concentration of AMG by newborn renal cortex. This indication that the tubule preparation reflects the in vivo situation led to an examination of the entry kinetics for AMG transport in isolated newborn cortical tubules. The results of this work constitute the basis for this report.

\section{MATERIALS AND METHODS}

\section{ANIMALS}

Female Sprague-Dawley rats were obtained from Charles River (Wilmington, MA) at 14 days of gestation and housed separately until parturition. Pups were killed by decapitation within the first $72 \mathrm{hr}$ of age. Adult male Sprague-Dawley rats, obtained from the same source and weighing 150-200 g, were used for comparison with the newborn.

\section{IN VIVO ACCUMULATION OF SUBSTRATE}

Newborn animals were injected intraperitoneally with $4 \mu \mathrm{l}$ of a 200 mM AMG (Pfanstiehl Co., Waukegan, IL.) solution containing $0.1 \mu \mathrm{Ci}{ }^{14} \mathrm{C}$-label (Calatomic, Los Angeles, CA). At 10 and 30 min after injection the animals were decapitated. The blood was collected in a heparinized capillary tube and the kidneys were quickly removed and placed in chilled Krebs-Ringer bicarbonate buffer, pH 7.4 (KRB). Since the blood volume of the newborn animal was so small the plasma was pooled from all animals killed at each time point. The kidneys from each animal were treated as one member of a group of values obtained at a respective time. Freehand slices of cortex were made, quickly blotted, and weighed. One milliliter of distilled water was added to all slices from each pair of kidneys and boiled for $3 \mathrm{~min}$. Aliquots of $20 \mu \mathrm{l}$ from the pooled deproteinized plasma plus $0.2 \mathrm{ml}$ of water were added to $1.5 \mathrm{ml}$ of Soluene (Lab Industries, Berkeley, CA), and $10 \mathrm{ml}$ of ACS fluid (Amersham-Searle, Arlington Heights, IL). Aliquots of $0.2 \mathrm{ml}$ from the tissue extracts were treated in a similar fashion. Plasma and tissue samples were counted in a Packard liquid scintillation spectrometer.

The "trapped medium" space and total tissue water of the newborn cortex slice has been previously reported from this laboratory (17) as $23 \%$ and $78.5 \%$, respectively. Corresponding values for the adult cortex slice are $25 \%$ and $80 \%$, respectively. Tissue AMG concentration and distribution ratios (ratio of $\mathrm{cpm} / \mathrm{ml}$ to intracellular fluid to $\mathrm{cpm} / \mathrm{ml}$ medium) were determined as described elsewhere (11).

Adult male animals, weighing $150-200 \mathrm{~g}$, were injected intraperitoneally with $100 \mu \mathrm{l}$ of $200 \mathrm{mM}$ AMG solution containing 0.1 $\mu \mathrm{Ci}$. These animals were killed by stunning and decapitation at the times mentioned above and the blood from each animal was collected into a heparinized syringe. The kidneys were quickly removed and placed in chilled KRB. Slices made from the kidneys of each animal were pooled, weighed, and treated as above. In this case, the deproteinized plasma from each animal was assayed for radioactivity and compared to the radioactivity found in the tissue from the same animal. Data derived from each animal handled in this fashion were treated as one member of a group for each time point.

\section{PREPARATION OF ISOLATED PROXIMAL TUBULES}

Tubules were prepared by a technique modified from that originally described by Burg and Orloff (4). After decapitation of six litters (40-60 animals), kidneys were removed immediately and placed in ice-cold KRB containing $10 \mathrm{mM}$ sodium acetate, $\mathrm{pH}$ 7.4. Cortical chips were cut in a freehand manner using a $\# 11$ scalpel blade. The chips were quickly minced and placed into 0.5 $\mathrm{ml} \mathrm{KRB}$ with $0.375 \%(\mathrm{w} / \mathrm{v})$ collagenase (grade II, Worthington Biochemical Corp., Freehold, NJ). 
After a 45-min digestion at room temperature, the tubules were resuspended in ice-cold KRB and centrifuged for $90 \mathrm{sec}$ at $40 \times$ $g$ in an International model UV centrifuge (this was repeated three times). The last suspension was then filtered through two layers of nylon stocking and brought to the desired final volume with KRB. Fetal calf serum (Flow Labs, Rockville, MD) was added, $1 \mathrm{ml}$ per $20 \mathrm{ml}$ final volume. Final suspensions contained $10-12 \mathrm{mg}$ of tubules per ml. Adult tubules were prepared from kidneys of 3-4 animals by the same method, except that the slices were made with a Stadie-Riggs microtome and the filtration step was carried out using three layers of surgical gauze instead of nylon stocking.

\section{DETERMINATION OF SUGAR ACCUMULATION}

Uptake studies were performed in adult and newborn tubule preparations simultaneously, in a manner identical to that previously described $(4,12,13)$. Distribution ratios $(\mathrm{cpm} / \mathrm{ml}$ intracellular fluid to $\mathrm{cpm} / \mathrm{ml}$ medium) were calculated by the method of Rosenberg et al. (11). All studies were carried out at $37^{\circ}$, using 0.1 $\mu \mathrm{Ci} / \mathrm{ml}$ of labeled $\alpha$-methyl-D-(U- $\left.{ }^{14} \mathrm{C}\right)$ glucoside $\quad(52.2$ $\mathrm{mCi} / \mathrm{mmole}$ ) (Calatomic, Los Angeles, CA) and unlabeled AMG (Pfanstiehl Co., Waukegan, IL) in sufficient quantities to give the desired final concentrations. Results were expressed as a plot of the distribution ratio vs. time of sampling. Because there is no metabolism of AMG by rat kidney cortex (17), distribution ratios represented a true gradient.

The volume of "trapped medium" in the pellet was established by the method of Burg and Orloff (4), using inulin-carboxyl- ${ }^{14} \mathrm{C}$ (New England Nuclear, Boston, MA). The volume of intracellular water was taken as the difference between total tissue water, determined by overnight dessication and the volume of trapped medium.

Studies of the concentration dependence of uptake for AMG were carried out at $37^{\circ}$. Incubations were carried out with continuous gassing and with various concentrations of unlabeled sugar ranging from $0.02 \mathrm{mM}$ to $5.0 \mathrm{mM}$, plus $0.1 \mu \mathrm{Ci} / \mathrm{ml}$ suspension of labeled AMG. All studies were continued for $5 \mathrm{~min}$, at which time aliquots were removed and treated as previously described (12). Glucose inhibition of AMG uptake was studied using the same technique. D-glucose (Pfanstiehl Co., Waukeegan, IL) was added to the experimental flasks in quantities sufficient to give the desired inhibitor concentration, and control flasks were run simultaneously without added glucose. Distribution ratios and velocities were calculated and standard Lineweaver-Burk plots made from which the apparent transport $\mathrm{K}_{\mathrm{m}}$ and $\mathrm{V}_{\max }$ were determined. Lines were fitted to the data points using a Monroe 1775 model programmed calculator, and results were analyzed by Student's $t$ test for statistical significance.

Other experiments were performed using the same basic technique, but with $\mathrm{AMG}$ at one very low concentration $(0.04 \mathrm{mM})$ and at a high concentration $(1.0 \mathrm{mM})$ in the presence of a variety of inhibitors. Phlorizin (Mann Research, New York, NY), ouabain (Sigma Chemical Corp., St. Louis, MO), 2,4-dinitrophenol and sodium fluoride (J. T. Baker, Phillipsburg, NJ), D-glucose, Dgalactose, and D-fructose (Pfanstiehl Co., Waukegan, IL), Tris

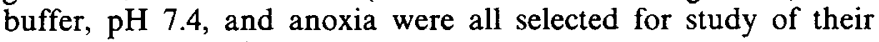
inhibitory properties.

\section{RESULTS}

\section{CONCENTRATIVE UPTAKE IN VIVO}

Injection of labeled $A M G$ solution into the newborn animal resulted in time-dependent accumulation of the substrate by the kidney. Tissue levels achieved in the adult at $10 \mathrm{~min}$ were significantly higher than those seen in the newborn, reflecting the differences in concentrating ability seen in vitro. Intracellular concentrations of AMG exceeding that of plasma were achieved in both newborn and adult cortex within $10 \mathrm{~min}$ of injection (Table 1). Plasma AMG concentrations are shown in Table 1, together with the simultaneous blood-glucose values. Urinary excretion of the isotope was not measured.

\section{CONCENTRATION-DEPENDENT UPTAKE IN VITRO}

Uptake of AMG by the newborn tubule occurs at a slow initial rate with the establishment of a low steady state concentration gradient as compared to previous reports for AMG uptake by the adult tubule $(12,13)$. Accumulation of the glucoside was studied for both newborn and adult tubules at two different concentrations $(0.2 \mathrm{mM}$ and $2.0 \mathrm{mM})$. These data are shown in Figure 1 .

The isolated adult tubule was found to have saturable uptake of AMG at concentrations above $0.2 \mathrm{mM}$ (see Fig. $2 D$ ). Reciprocal plots of velocity determinations at concentrations from 0.02 to 0.2 $\mathrm{mM}$ AMG show a line intersecting the origin with increasing substrate concentration, suggesting the absence of saturable uptake in this concentration range. Cortical slices of adult rat kidney behave in a similar fashion in this concentration range (17). In

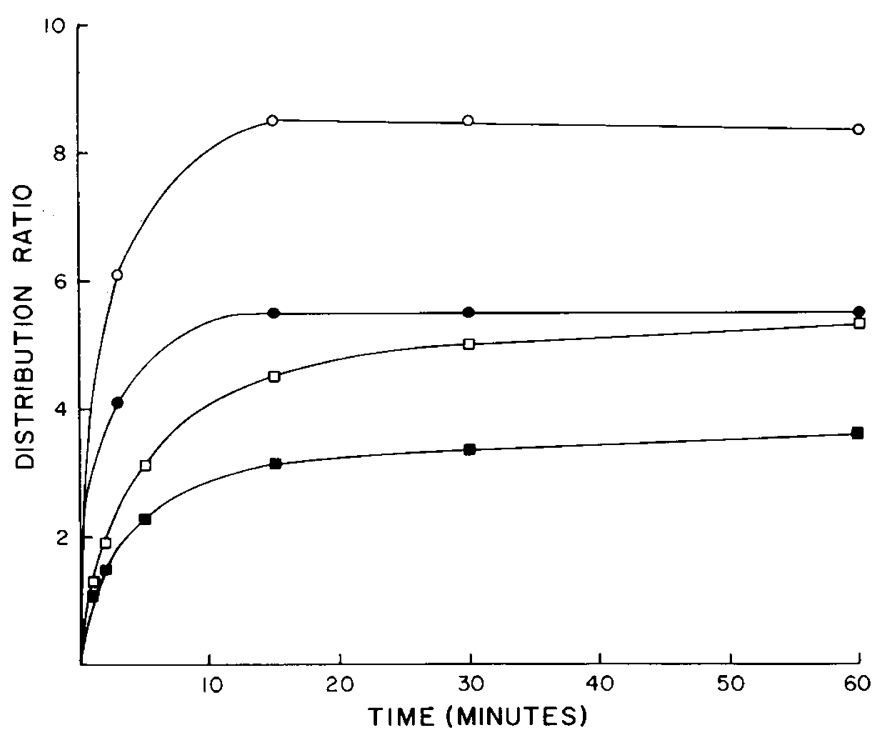

Fig. 1. Saturable uptake by newborn and adult isolated renal tubules. Uptake by newborn isolated tubules at $2 \mathrm{mM}(\mathbb{0})$ and $0.2 \mathrm{mM}(\square)$ is compared to uptake by isolated adult tubules at $2 \mathrm{mM}(\bullet)$ and $0.2 \mathrm{mM}$ (O) AMG. Conditions are described in text. Each curve represents the mean of at least triplicate experiments. Distribution ratio is the ratio of $\mathrm{cpm} / \mathrm{ml}$ of intracellular fluid to $\mathrm{cpm} / \mathrm{ml}$ of medium.

Table 1. Uptake of $A M G{ }^{14} \mathrm{C}$ by adult and newborn rat kidney cortex in vivo ${ }^{1}$

\begin{tabular}{llclll}
\hline & \multicolumn{2}{c}{ Plasma AMG concentration (mM) } & & \multicolumn{2}{c}{ Kidney distribution ratio } \\
\cline { 2 - 3 } \cline { 5 - 6 } & \multicolumn{1}{c}{$10 \mathrm{~min}$} & $30 \mathrm{~min}$ & & $10 \mathrm{~min}$ & $30 \mathrm{~min}$ \\
\hline Newborn & 0.198 & 0.141 & & $1.492 \pm 0.196$ & $3.641 \pm 0.210$ \\
Adult & $0.207 \pm 0.010$ & $0.303 \pm 0.011$ & & $5.723 \pm 0.383$ & $4.600 \pm 0.241$ \\
\hline
\end{tabular}

${ }^{1}$ Mature Sprague-Dawley male rats (150-200 g) and newborn animals of both sexes ( $<72 \mathrm{hr}$ old) were used in these experiments of which the data presented is a representative sample. The techniques used for injection and sampling are described in text. Each value represents the mean ( \pm standard error) of data obtained from at least three animals (no standard error is given for the newborn plasma concentration because the blood was a pooled sample). Blood glucose in the newborn was $81 \mathrm{mg} / \mathrm{dl}$ and in the adult was $127 \mathrm{mg} / \mathrm{dl}$, determined by a Beckman model ERA 2001 glucose analyzer. 


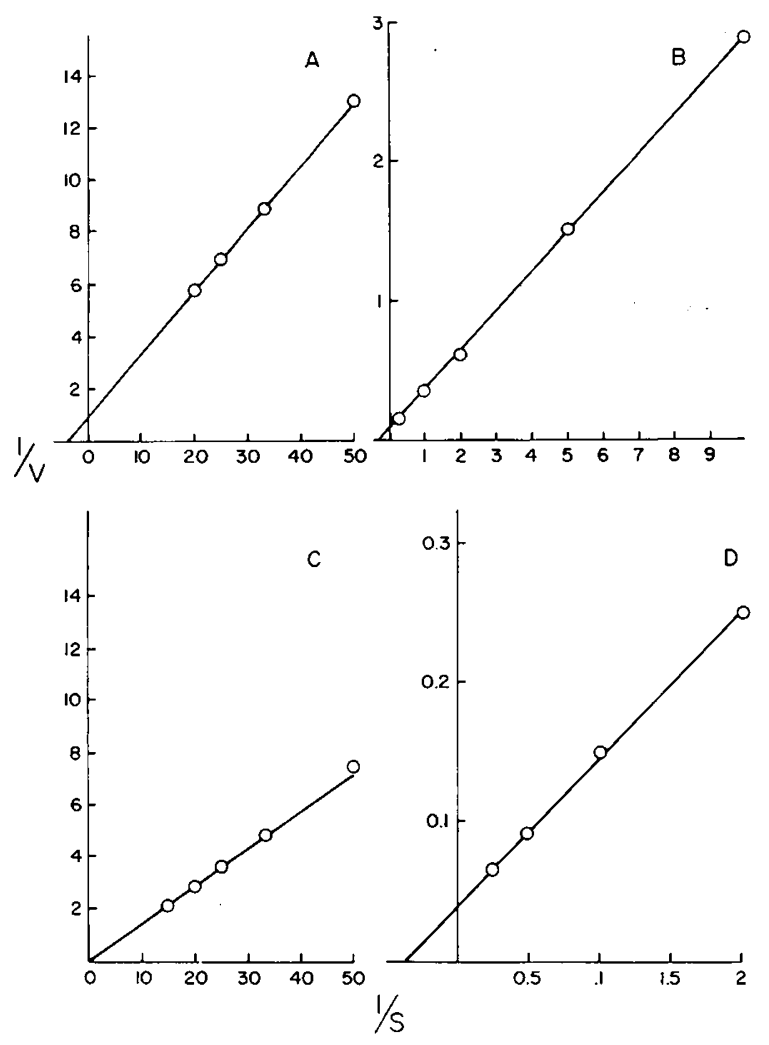

Fig. 2. Reciprocal plots of concentration-dependent uptake of AMG by newborn $(A$ and $B)$ and adult $(C$ and $D$ ) isolated renal tubules. Conditions are described in text. The reciprocal values of $\mathrm{V}(5$-min velocity of uptake in mmols/liter of intracellular water) against $1 / \mathrm{S}$ (the reciprocal values of their respective substrate concentration in mmols/liter) are shown. All points represent the mean of at least triplicate experiments. Correlation factors for the lines shown were 0.998 , and the lines differ for the adult and newborn $(P<0.001)$.

contrast to both the adult tubule and slice, the isolated newborn tubule showed concentration-dependent uptake over the entire substrate concentration range from 0.02 to $5.0 \mathrm{mM}$ (Fig. $2 A$ and $2 B$ ). Reciprocal plots of these data revealed two distinct lines, one of which had a calculated $\mathrm{K}_{\mathrm{m}}$ identical to that observed in the adult at substrate concentrations above $0.2 \mathrm{mM}$. At concentrations below $0.2 \mathrm{mM}$, the velocity data clearly described a second line which was interpreted to represent a second transport system in the newborn (Fig. 2A). Subtraction of a diffusion component determined according to the method of Akedo and Christensen (1) did not alter the results as stated. The $V_{\max }$ and $K_{m}$ parameters for both adult and newborn tubules are shown in Table 2. Statistical analysis of the velocity data confirmed the presence of two systems in both adult and newborn tubules $(P<0.001)$.

\section{EFFECT OF INHIBITORS ON CONCENTRATION-DEPENDENT UPTAKE}

Further characterization of the transport systems was undertaken, using various inhibitors. Uptake of AMG by adult and newborn tubules was studied in the presence of D-glucose, a known competitive inhibitor of AMG uptake in the adult rat cortical slice (17). Inhibition of AMG uptake at substrate concentrations above $0.5 \mathrm{mM}$ was competitive in both preparations. The $\mathrm{K}_{\mathrm{i}}$ for D-glucose in the newborn tubule was $1.81 \mathrm{mM}$ and in the adult tubule $1.4 \mathrm{mM}$, thus emphasizing the fact that the newborn and adult kidney share the low affinity transport system.

Utilizing the arrangement of the Michaelis-Menten formula for transport by multiple systems:

$$
\mathrm{V}=\mathrm{V}_{\max 1}(\mathrm{~S}) /\left[\mathrm{K}_{\mathrm{m} 1}+(\mathrm{S})\right]+\mathrm{V}_{\max 2}(\mathrm{~S}) /\left[\mathrm{K}_{\mathrm{m} 2}+(\mathrm{S})\right]
$$

it was possible to calculate that at AMG concentrations as low as $0.01 \mathrm{mM}$ the high affinity system in the newborn contributed only
$50 \%$ of total uptake. Hence, it was not possible to examine each transport system independent of the other. This difficulty was emphasized by a failure to obtain consistent results in examining the high affinity system of the newborn in the presence of $D$ glucose.

The effects of various inhibitors on the transport of AMG by both newborn and adult tubules are shown in Table 3. At substrate concentrations of $0.04 \mathrm{mM}, 46 \%$ of the newborn uptake occurs on the high affinity system. At $1.0 \mathrm{mM} \mathrm{AMG}$, only $20 \%$ of newborn uptake is accounted for by this system, the remaining $80 \%$ taking place by means of the low affinity system which is present in the adult tubule as well. Comparing the response to inhibitors of this latter system in the adult to that of the newborn, it is obvious from Table 3 that both respond very similarly. Exceptions to this occur in the presence of $10 \mathrm{mM}$ fructose and 2,4-dinitrophenol, neither of which affect the newborn high $\mathrm{K}_{\mathrm{m}}$ system. On the other hand, when $46 \%$ of total uptake occurred on the low $\mathrm{K}_{\mathrm{m}}$ system in the newborn (at $0.04 \mathrm{mM}$ AMG), anaerobiosis was the only potential inhibitor tested which had no significant effect and was different from its effect on the adult system.

Although AMG transport was significantly reduced in both the newborn and the adult tubule in the presence of sodium-free Tris buffer, each retained a degree of concentrative uptake which exceeded that observed in the presence of phlorizin. The effect of $1.0 \mathrm{mM}$ ouabain was similar to that of Tris buffer. Sodium fluoride did not affect transport of AMG by either the newborn or the adult tubule at either substrate concentration.

\section{DISCUSSION}

Active accumulation of sugars has not been previously demonstrated in newborn rat kidney cortical slice $(2,16,17)$, although neonatal rat diaphragm (7) and small intestine (6) have been shown to concentiate various hexoses. A recent report (13), however, has shown that the isolated proximal tubule fragment from newborn Sprague-Dawley rat kidney is capable of concentrative uptake of AMG. This observation has now been found to be true of the newborn kidney in vivo as well, thus confirming the newborn isolated tubule fragment to be a valid model system for the study of sugar transport in vitro.

Because uptake studies at substrate concentrations varying 20 fold showed a consistently slower initial rate of uptake by newborn tubules as compared to the adult, the likelihood existed that there were differences in entry rate kinetics to account for the observed rate in the immature tissue. Analysis of the entry phenomenon in both newborn and adult tubules confirmed this possibility. The newborn tubules were found to have two membrane transport systems for AMG differing 18-fold in their apparent transport $\mathrm{K}_{m}$ value as compared to the adult tubule which has a saturable and a nonsaturable system. Numerically, the values for the apparent transport $\mathrm{K}_{\mathrm{m}}$ in the adult agrees well with previous reports in adult tubule (12) and adult renal cortical slice (17). Furthermore, there is excellent agreement between the values observed for the low affinity system in the newborn and saturable system in the adult, suggesting that this system might be shared by mature and immature tissue. Uptake on this system was competitively inhibited by D-glucose, yielding the same $K_{i}$ value in both newborn and adult tubules, and anaerobiosis significantly decreased accu-

Table 2. Kinetic parameters of concentration-dependent uptake of AMG by newborn and adult isolated renal tubules ${ }^{1}$

\begin{tabular}{lccr}
\hline & & Newborn & Adult \\
\hline $\mathrm{K}_{\mathrm{m}}(\mathrm{mM})$ & 0.244 & 4.082 & 4.341 \\
$\mathrm{~V}_{\max }$ (mmols/liter intracel- & 1.005 & 17.026 & 32.035
\end{tabular}

lular fluid $/ 5 \mathrm{~min}$ )

${ }^{1}$ All experiments were performed at $37^{\circ}$ for $5 \mathrm{~min}$. The values shown represent the means of at least three experiments. For procedural details, see text. The $\mathrm{K}_{\mathrm{m}}$ values for the newborn are significantly different $(P<$ $0.001)$. 
Table 3. Effect of various inhibitors on accumulation of $A M G$ by isolated adult and newborn rat tubules ${ }^{1}$

\begin{tabular}{|c|c|c|c|c|}
\hline \multirow[b]{3}{*}{ Inhibitor (concentration) } & \multicolumn{4}{|c|}{ Distribution ratio } \\
\hline & \multicolumn{2}{|c|}{$0.04 \mathrm{mM}$ substrate } & \multicolumn{2}{|c|}{$1.0 \mathrm{mM}$ substrate } \\
\hline & Newborn & Adult & Newborn & Adult \\
\hline Control & $3.482 \pm 0.108(20)$ & $6.448 \pm 0.590(11)$ & $2.891 \pm 0.638(20)$ & $5.922 \pm 0.340(12)$ \\
\hline Phlorizin $(0.5 \mathrm{mM})$ & $0.248 \pm 0.010^{2}(6)$ & $0.597 \pm 0.014^{2}(3)$ & $0.225 \pm 0.019^{2}(6)$ & $0.554 \pm 0.035^{2}(3)$ \\
\hline Anaerobiosis & $3.494 \pm 0.484^{3}(6)$ & $5.136 \pm 0.212^{2}(5)$ & $2.076 \pm 0.056^{2}(6)$ & $4.213 \pm 0.213^{2}(5)$ \\
\hline Fructose $(10 \mathrm{mM})$ & $3.129 \pm 0.090^{2}(10)$ & $4.480 \pm 0.161^{2}(3)$ & $2.819 \pm 0.104^{3}(10)$ & $3.269 \pm 0.041^{2}(3)$ \\
\hline Glucose $(0.5 \mathrm{mM})$ & $2.793 \pm 0.314^{2}(6)$ & $3.829 \pm 0.175^{2}(3)$ & $2.222 \pm 0.188^{2}(6)$ & $4.129 \pm 0.066^{2}(3)$ \\
\hline Glucose $(1.0 \mathrm{mM})$ & $1.914 \pm 0.066^{2}(8)$ & $3.347 \pm 0.135^{2}(3)$ & $1.743 \pm 0.105^{2}(8)$ & $3.280 \pm 0.036^{2}(3)$ \\
\hline Galactose (10 mM) & $2.060 \pm 0.081^{2}(10)$ & $3.995 \pm 0.163^{2}(3)$ & $1.783 \pm 0.063^{2}(10)$ & $3.893 \pm 0.083^{2}(3)$ \\
\hline Ouabain (1.0 mM) & $1.397 \pm 0.033^{2}(6)$ & $2.638 \pm 0.078^{2}(3)$ & $1.228 \pm 0.032^{2}(6)$ & $2.581 \pm 0.074^{2}(3)$ \\
\hline $\mathrm{Na}^{+}$-free Tris, $\mathrm{pH} 7.4$ & $0.523 \pm 0.026^{2}(6)$ & $2.193 \pm 0.103^{2}(6)$ & $0.446 \pm 0.013^{2}(6)$ & $2.177 \pm 0.199^{2}(6)$ \\
\hline $\mathrm{NaF}(10 \mathrm{mM})$ & $3.558 \pm 0.138^{3}(6)$ & $7.816 \pm 0.518^{3}(6)$ & $3.364 \pm 0.077^{3}(6)$ & $6.026 \pm 0.393^{3}(6)$ \\
\hline $2,4-\mathrm{DNP}(0.12 \mathrm{mM})$ & $2.671 \pm 0.063^{2}(6)$ & $3.009 \pm 0.041^{2}(3)$ & $2.716 \pm 0.065^{3}(6)$ & $3.390 \pm 0.150^{2}(3)$ \\
\hline
\end{tabular}

${ }^{1}$ Incubation was carried out for $5 \mathrm{~min}$ at $37^{\circ}$ as described in text. The procedure for the determination of the distribution ratio, the ratio of cpm/ml intracellular water to $\mathrm{cpm} / \mathrm{ml}$ medium, is described in text. Mean $\pm \mathrm{SE}$ is given with number of analyses in parentheses.

${ }^{2} P<0.05$ to 0.001 .

${ }^{3} P>0.05$.

mulation in both. These observations tend to confirm the possibility that this low affinity system previously reported to be present in adult isolated tubules (12) is also present in the immediate newborn period, although functioning less efficiently.

Multiple carrier transport of hexoses in rat kidney cortex in vitro has not been previously reported, although such a phenomenon has been described in hamster (8) and rat (3) small intestine. Although the kinetic analysis shows a two-limbed curve for the newborn tubule which is distinctly different from that of the adult, it was not possible to study the high affinity system in isolation because substrate concentrations as low as $10^{-3} \mathrm{mM}$ could not restrict the contribution of the low affinity system to less than $50 \%$. At $0.04 \mathrm{mM}$ AMG, when the high affinity system is contributing $46 \%$ of the total, newborn transport was affected by anaerobiosis, $10 \mathrm{mM}$ fructose, and 2,4-DNP in a manner different from the effects of these inhibitors on transport at $1.0 \mathrm{mM}$ AMG. Although we can only speculate as to the biochemical mechanisms involved, these data clearly support the presence of a second transport system in the newborn. On the other hand, the adult tubule responded to all inhibitors tested in an identical manner irrespective of substrate concentration, signifying that uptake at both high and low AMG concentrations occurred on the same system.

Silverman (19) has claimed that AMG transport in the dog kidney is exclusively a brush-border phenomenon, with efflux also possible only at the brush-border surface. If this is true for the rat kidney as well, then data presented in this report must describe the entry phenomenon at the tubule brush-border surface. The finding that neither sodium-free medium nor $1.0 \mathrm{mM}$ ouabain totally inhibited transport of AMG is in agreement with the data of Kinne $e t$ al. who showed that D-glucose transport by antiluminal membrane vesicles from rat kidney is relatively independent of $\mathrm{Na}^{+}$-ion concentration and of lower capacity than the corresponding transport system on the brush-border surface (9). This would suggest that a portion of the transport observed under these conditions in the tubules is antiluminal in nature. In either case, the significance of these observations is that the greatest component of the transport of AMG by the isolated tubule is occurring across the luminal surface. Furthermore, Silverman's work (18) showing that the D-glucose transport protein in the dog is identical to the AMG carrier suggests that the present observations may be completely analogous to events taking place during luminal glucose transport in the rat kidney.

Christopher et al. (5) have published a report describing multiple carrier transport in chick embryo fibroblasts. In this report they describe a repression-depression phenomenon related directly to the metabolic state of the cells. In the sugar-depleted state, the cells appeared to develop a high affinity system for D-glucose transport which disappeared when the cells were fed. The development of this high affinity transport system could be inhibited by $N$-ethylmaleimide or cycloheximide, suggesting that the phenomenon actually depended upon protein synthesis. The authors speculate on the survival advantage conferred upon a cell which has the capability for enhancing its ability to take up substrate which is in short supply. Such a capability for enhanced uptake has also been demonstrated in newborn kidney cortex slices by Reynolds and Segal (10), although this work dealt with amino acids and concluded that the enhanced ability for uptake was related to an increased $V_{\max }$, rather than to a new species of carrier protein. In earlier work (14), the existence of a high affinity transport system for glycine was demonstrated in the newborn rat kidney tubule. This system diminished in importance with age, until in the adult tubule virtually no transport occurred on the high affinity system at physiologic glycine concentrations. This same situation appears to pertain in the present report for AMG transport. All of these findings would suggest that high affinity transport systems are more characteristic of the immature tissue of at least two species than of the adult tissue.

\section{CONCLUSION}

AMG is accumulated against a chemical gradient by newborn rat kidney cortex in vivo and in vitro. This uptake occurs by means of two systems: 1) a high $\mathrm{K}_{\mathrm{m}}$ system which has kinetic and inhibitory properties which are shared by a similar system in adult rat kidney, and 2) a low $\mathrm{K}_{\mathrm{m}}$ system with a very high affinity for AMG which is unique to the newborn. These observations form the basis for a possible explanation of the absence of glycosuria in the newborn rat, a phenomenon which has hitherto remained unclarified.

\section{REFERENCES AND NOTES}

1. Akedo, H., and Christensen, H. N.: Nature of insulin action on amino acid uptake by the isolated diaphragm. J. Biol. Chem., 237: 113 (1962).

2. Bailey, J. M., Fishman, P. H., and Pentchev, P. G.: Studies on mutarotases. VI. Enzyme levels and sugar reabsorption in developing rat kidney and intestine. J. Biol. Chem., 245: 559 (1970).

3. Berman, W. G., Bautista, J. O., Rogers, S., and Segal, S.: Metabolism and transport of galactose by rat intestine. Biochim. Biophys. Acta, 455: 90 (1976).

4. Burg, M. B., and Orloff, J.: Oxygen consumption and active transport in separated renal tubules. Am. J. Physiol., 203: 327 (1962).

5. Christopher, C. W., Kohlbacher, M. S., and Amos, H.: Transport of sugars.in chick-embryo fibroblasts: Evidence for a low-affinity system and a high-affinity system for glucose transport. Biochem. J., I58: 439 (1976).

6. Fitzgerald, J. F., Reiser, S., and Christiansen, P. A.: Developmental pattern of sugar and amino acid transport in the postnatal rat small intestine. Pediatr. Res., 5: 698 (1971).

7. Fricke, R., and Clark, C. M., Jr.: Augmentation of glucose and amino acid uptake by insulin in the developing rat diaphragm. Am. J. Physiol., 224: 117 (1973). 
8. Honegger, P., and Semenza, G.: Multiplicity of carriers for free glucalogues in hamster small intestine. Biochim. Biophys. Acta, 318: 390 (1973).

9. Kinne, R., Murer, H., Kinne-Saffran, E., Thees, M., and Sachs, G.: Sugar transport by renal plasma membrane vesicles: characterization of systems in the brush-border microvilli and basal-lateral plasma membranes. J. Membr. Biol., 21: 375 (1975).

10. Reynolds, R., and Segal, S.: Regulatory characteristics of amino acid transport in newborn rat renal cortex cell. Biochim. Biophys. Acta, 426 : 513 (1976).

11. Rosenberg, L. E., Blair, A., and Segal, S.: The transport of amino acids by rat kidney cortex slices. Biochim. Biophys. Acta, 54: 179 (1961).

12. Roth, K. S., Hwang, S. M., and Segal, S.: The effect of maleic acid on the kinetics of alpha-methyl-D-glucoside uptake by isolated rat renal tubules. Biochim. Biophys. Acta, 426: 675 (1976).

13. Roth, J. S., Hwang, S. M., Yudkoff, M., and Segal, S.: On the transport of sugars and amino acids by newborn kidney: Use of isolated proximal tubule. Life Sci., I8: 1125 (1976)

14. Roth, K. S., Hwang, S. M., London, J., and Segal, S.: Ontogeny of glycine transport in isolated rat renal tubules. Am. J. Physiol., 233: F241 (1977).

15. Segal, S., Genel, M., and Smith, I.: The effect of storage on alpha-methyl-Dglucoside transport by rat kidney cortex slices. J. Lab. Clin. Med., 72: 778 (1968).

16. Segal, S., Rea, C., and Smith, I.: Separate transport systems for sugars and amino

Copyright (C) 1978 International Pediatric Research Foundation, Inc $0031-3998 / 78 / 1212-1127 \$ 02.00 / 0$ acids in developing rat kidney cortex. Proc. Natl. Acad. Sci. (U.S.A.), 68: 372 (1971).

17. Segal, S., Rosenhagen, M., and Rea, C.: Developmental and other characteristics of alpha-methyl-D-glucoside transport by rat kidney cortex slices. Biochim. Biophys. Acta, 291: (1973).

18. Silverman, $\mathbf{M}$, and Black, $\mathbf{J}$ : High affinity phlorizin receptor sites and their relation to the glucose transport mechanism in the proximal tubule of the dog kidney. Biochim. Biophys. Acta, 394: 10 (1975).

19. Silverman, M: Glucose transport in the kidney. Biochim. Biophys. Acta, 457: 303 (1976).

20. This work was presented, in part, at the meetings of the Society for Pediatric Research, April 1977, San Francisco, California.

21. This research was supported by Grant AM 10894 and Training Grant HD 00215 from the National Institutes of Health.

22. Dr. Karl S. Roth is the recipient of a Daland Foundation Fellowship from the American Philosophical Society.

23. Requests for reprints should be addressed to: Dr. Stanton Segal, Department of Pediatrics, University of Pennsylvania School of Medicine, Children's Hospital of Philadelphia, One Children's Centre, 34th and Civic Centre Boulevard, Philadelphia, PA 19104 (USA).

24. Received for publication June 30, 1977

25. Accepted for publication February 8, 1978. 